Document downloaded from:

http://hdl.handle.net/10251/63327

This paper must be cited as:

Andres, B.; Poler, R.; Sanchis, R. (2015). Collaborative Strategies Alignment to Enhance the Collaborative Network Agility and Resilience. IFIP Advances in information and communication technology. 463:88-99. doi:10.1007/978-3-319-24141-8.

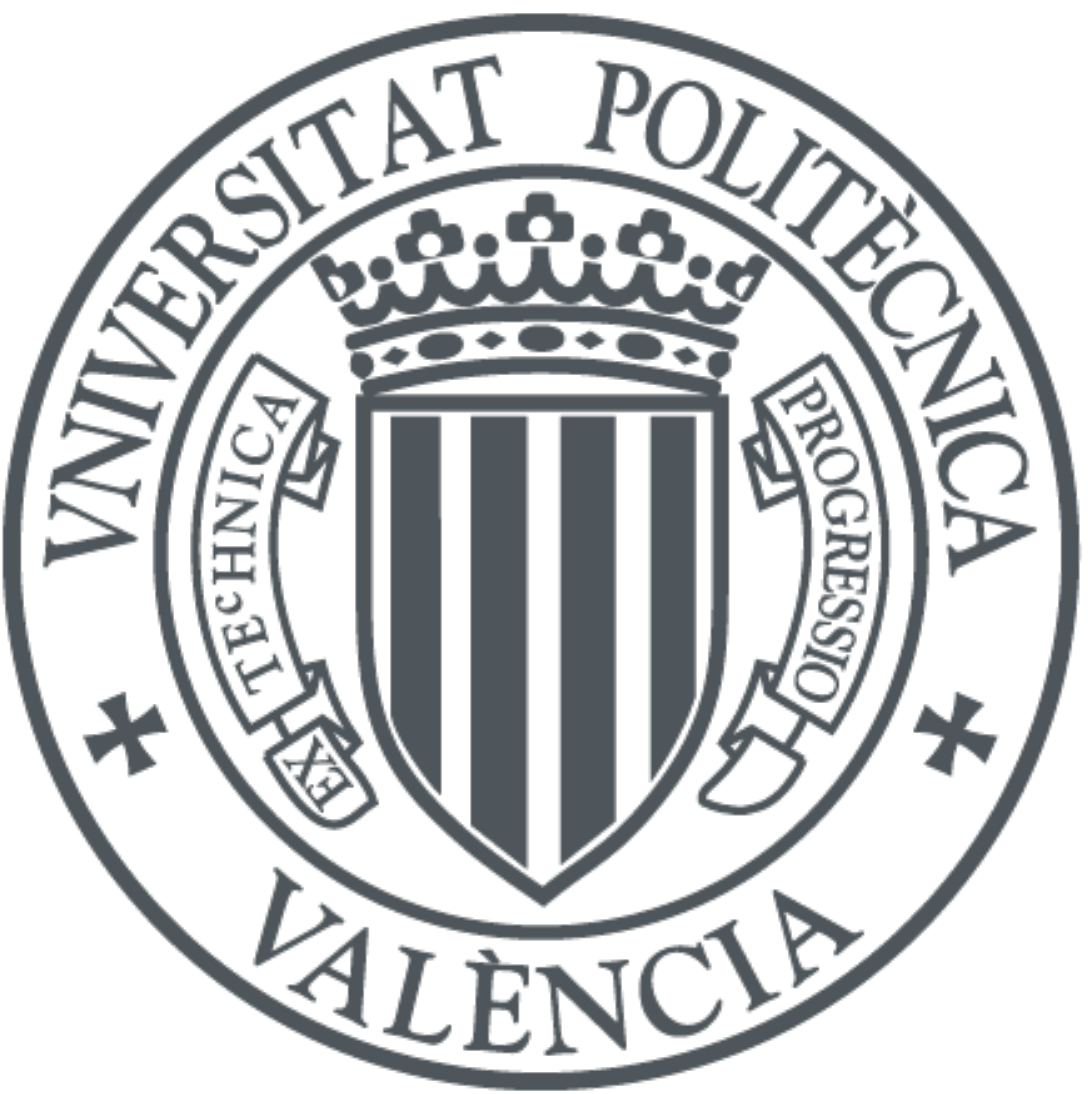

The final publication is available at

http://dx.doi.org/10.1007/978-3-319-24141-8_8

Copyright Springer

Additional Information 


\title{
Collaborative Strategies Alignment to Enhance the Collaborative Network Agility and Resilience
}

\author{
Beatriz Andres ${ }^{1}$, Raul Poler ${ }^{1}$, Raquel Sanchis ${ }^{1}$ \\ 1 Research Centre on Production Management and Engineering (CIGIP). Escuela Politécnica \\ Superior de Alcoy, Universitat Politècnica de València (UPV). Centre d'Innovació i \\ Investigació. Calle Alarcón, 03801 Alcoy, Spain \\ \{bandres, rpoler, rsanchis\}@ cigip.upv.es
}

\begin{abstract}
Current supply networks are embedded in dynamic and turbulent environments, and must face the appearance of some disruptive events throughout their life cycle. Disruptions are almost always accompanied by negative effects, resulting on performance loss for both, the enterprises and the network. The activation of proactive strategies will allow enterprises to reduce this loss when a disruption appears. Enterprises must be aware of that the activated strategies must be aligned, so that they positively influence the objectives and the performance indicators defined by all the network partners. This paper proposes a simulation model to support enterprises in the decisionmaking on which proactive strategies activate in order to be aligned, from a collaborative perspective. The main aim is to limite the adverse effects produced by the disruptions. Such aligned strategies allow collaborative enterprises to move in the same direction when a disruption appears. In addition the strategies enhance the resilience and agility of both the enterprises and the network as well as positively influence the objectives and the performance indicators defined by all the network partners
\end{abstract}

Keywords: Strategies Alignment, Disruptions, Collaborative Processes, Process Disruptions, System Dynamics, Proactive Actions, Resilience

\section{Introduction}

The current global business environment, characterised by being unpredictable and competitive, makes enterprises to be more exposed to disruptive events. This encourages enterprises to change the way they work and to be more flexible in the process of recovering themselves from disruptions. Thus, a new tendency is emerging through the enterprises participation in Collaborative Networks (CN) [1]. Such participation requires to restructure their internal operations, make information systems interoperable, coordinate their production processes, align their strategies, share goals, achieve suitable levels of trust, reach agreements in practices, and align values [2][3][4]. The benefits specifically associated with the strategies alignment have a great influence on the $\mathrm{CN}$ success, since they are becoming a relevant issue for achieving competitive advantages [4]. The participation in CNs allows enterprises to be more agile and resilient, and to increase the effectiveness in response to the effects 
of potential disruptive events [5]. Focusing on the strategies alignment process, carried out from a collaborative perspective, Andres and Poler [6] consider in their approach the strategies alignment to facilitate some of the conventional disruptions [7], such as the variability of networks, global competition, complexity in supply chain and greater variety in production.

When a disruption takes place, various independent enterprises are affected and each one defines a set of strategies to deal with the negative repercussions that impact its performance. These strategies can be proactively or reactively deployed [8]. Mitroff y Alpasan [9] stated that resilient organizations are proactive and they recover sooner and better from the disruptions. The decision of which proactive strategies to activate, in order to deal with the disruptions, can be made from a collaborative or non-collaborative perspective. This paper focuses on the proposal of a collaborative and proactive solution through the Strategies Alignment Simulation Model (SASM). SASM models the influences expected among the collaborative enterprises taking into account the objectives defined and the proactive strategies formulated by all the networked enterprises, modelling the influences exerted among them. The model is based on the System Dynamics (SD) method and promotes the activation of those proactive strategies that, being aligned, positively influence all objectives defined by all the network partners; enabling them to reduce the loss of business performance, after a disruptive event occurs.

In the light of this, the next research question appears:

What would be an adequate model to support enterprises in the decision making process of selecting proactive strategies to be aligned, in order to efficiently deal with the unexpected disruptive events, from a collaborative perspective?

The paper is organised as follows: Section 2 introduces the concept of disruption in supply networks as define in the literature. In section 3, the Strategies Alignment Simulation Model (SASM) is presented. A numerical example is described in section 4, applying the model to deal with a supply disruption. Finally in section 5, conclusions and future research lines are considered.

\section{Supply Networks Disruptions}

The term disruption, outlined by Barroso et al. [10], is defined as a predictable, or in most cases unpredictable event that directly affects the common activity and stability of an enterprise, thereby its performance. Sheffi and Rice [7] model the loss of business performance, defining 8 phases that enterprises experience when a disruption occurs (see Fig. 1): (i) preparation: companies anticipation and proactive attitude, (ii) disruptive event: any situation that threatens the daily operation of a company, (iii) first response: decision after reaction, (iv) initial impact: immediately disruption repercussion, (v) total impact: medium or long term effects (once the disruption occurs, the performance decreases significantly), (vi) preparation for recovery: starts in parallel with the first response, (vii) recovery: the stage in which the company returns to the state before the disruption and (viii) long-term impact: the time companies need, after a disruptive event, to recover (depending on the severity of the consequences). 


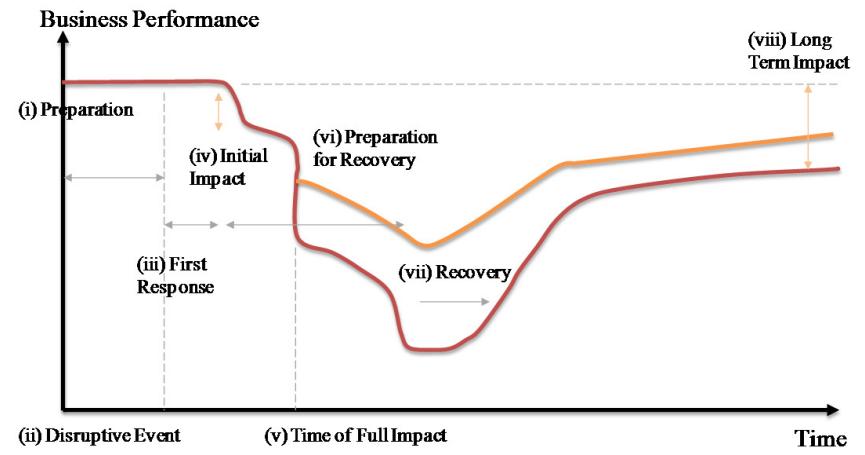

Fig. 1. Disruptions Phases (Adapted from Sheffi and Rice, 2005)

Work that can be highlighted in the scope of supply network disruptions are: Wu et al. [11] Disruption Analysis Network (DA_NET), determines how disruptions propagate and affect supply networks, through the methodology. Sheffi and Rice [7] focus their work on the disruptions classification so that can be easily identified and overcame. Ivanov et al. [12] focus on the potential disruptions identification using the Supply Chain Events Management (SCEM) or Sanchis and Poler [13] propose a categorization framework of disruptions as a starting point to evaluate the resilience capacity of enterprises.

\section{Strategies Alignment to Deal with Supply Disruptions}

In order to reduce the performance loss and be more resilient against any disruption, enterprises are encouraged to collaborate, and more concretely, to collaboratively align their strategies [6][14]. Therefore, a simulation model to collaboratively carry out the strategies alignment process is developed. The strategies alignment model is designed based on the assumption that the networked partners individually formulate a set of proactive strategies to manage the appearance of potential disruptions. Thus, it is crucial to collaboratively work to select those proactive strategies that are aligned, allowing the network members to reduce the negative influences reflected in the Business Performance (Key Performance Indicators, KPIs), when a disruptive event occurs (Fig. 1, orange line). The SASM supports enterprises in the collaborative decision making process of which strategies to activate in order to align their own strategies with the strategies formulated by all the partners of the network.

\subsection{Strategies Alignment Concept}

In order to give the reader a better understanding of how the strategies alignment process is treated in this paper, a definition of the concept strategies alignment is hereafter presented. 
It is widely known that the strategies are the set of actions raised to achieve the defined objectives, i.e. minimise the performance loss derived from a disruption by reducing the recovery time or cost, etc. Considering the work developed in [15], it is assumed that the strategies alignment concept is defined as, the set of strategies, formulated by all the network enterprises, whose activation positively influence the achievement of all the objectives defined by all the partners, increasing their KPI levels. The activation of the aligned strategies will promote the maximization of the positive influences and the minimization of the negative influences, respectively at the network level. Note: the concept of alignment is not the same as compatibility. Strategies are compatible when they can be activated at the same time but do not have positive influences on each other. The total benefit corresponds to the sum of the benefit obtained by the activation of each strategy individually. Lets consider two enterprises $e_{i}$ and $e_{j}$, each one defines one objective $o_{i}$ and $o_{j}$ and formulates one strategy, $S_{i}$ and $S_{j} . S_{i}$ and $S_{j}$ are considered to be aligned when the activation of $S_{i}$ has a positive influence on both objectives $o_{i}$ and $o_{j}$ and the same occurs with $S_{j}$. The strategies are aligned when the total benefit obtained is higher than the sum of the benefits obtained by the activation of each strategy individually. The strategies alignment concept is mathematically described in [4].

\subsection{Strategies Alignment Simulation Model (SASM)}

Despite the importance of aligning strategies in a $\mathrm{CN}$, in terms of avoiding partnership conflicts and moving in the same direction when dealing with disruptive events, a gap has been found in the literature with respect to the contributions of a holistic approach. An approach that considers all the strategies formulated by all the partners, when deciding which aligned strategies to activate in order deal with a disruption. To fill this gap, this research aims to support the decision making process of identifying which of the proactive strategies have to be activated, by a simulation model. The holistic perspective, will allow modelling the strategies alignment process regardless the strategies' nature and the type of disruption facing, considering the $\mathrm{CN}$ context. The proposed SASM allows the modelling the CN considering the elements that define the strategies alignment process. These elements and the relations established among them are represented through a causal and flow chart according to the SD Method [16]. For the model formulation, the following considerations have been taken into account:

- Each networked enterprise defines a set of objectives, which will be measured before and after a disruption occurs. The extent into which the objectives are achieved is measured through KPIs. The enterprises' aim is to achieve the maximum level of each KPI (what means to minimise the performance reduction), and to obtain, as fast and at lowest costly possible, the maximum levels of network performance in the recovery phase and in the long term, once a potential disruptive event occurs.

- A set of proactive strategies is formulated by each network enterprise, with the main objective of dealing with future potential disruptions and minimise the performance loss of the defined objectives (KPIs). The strategies are devoted to 
improve the performance level of each KPI, and consequently to improve the network performance.

- The use of KPIs allows computing the increase/decrease of the network and enterprises performance when a specific set of strategies is activated.

- Not all the proactive strategies formulated will be activated; the enterprises will only carry out those that are aligned.

The SASM will allow analysing, describing, explaining, simulating, assessing, monitoring and predicting misalignments among the strategies specifically those formulated to deal with disruptions. Moreover, the simulation model is proposed as a supporting tool for the enterprise decision makers, to identify which proactive strategies to activate in order to obtain higher levels of alignment, and consequently of performance, not only at the intra-enterprise level but also with the rest of enterprises of the network, that is the inter-enterprise level. In the light of this, recovery time and cost will be reduced, increasing the levels of resilience of both the enterprises and the network.

System Dynamics Method (SD). Solving the strategies alignment model through analytical methods implies to face tedious procedures involving large number of iterations. For that reason the use of the SD method is considered. SD method, outlined by Forrester [16], allows to analyse the characteristics of the feedbacks of the represented system $(\mathrm{CN})$, allowing to understand how the elements, belonging to the system, interact with each other, influencing in its performance. Generally, SD allows to understand the causal relationships of systems' behaviour by bringing together sets of elements that are interrelated in such a way that a change in one element affects a whole series of elements [17]. In the context of strategies alignment, SD allows representing the influences that the activated strategies have on the KPIs level. The relations of influences are represented in the SASM. Depending on the strategies activated, the KPIs level will be positively or negatively influenced. In the particular scenario considered in this paper, the SD method allows to simulate all the elements of the system (proactive strategies formulated, objectives defined and their relations) by simultaneously changing the decision variables, which are defined by (i) the units of strategies to activate ( $u_{-}$Sis) and (ii) the time when to activate them (ti_Sis). This will enable the SASM to identify the proactive strategies appropriate to be activated, so that positive influences are obtained in all the KPIs defined by all the networked enterprises

The causal loop diagram allows representing the elements and relationships of the modelled system, based on an influence on effects (+ and - loops) (Campuzano and Mula, 2011) [17]. The flow diagram translates the information depicted in the causal loop diagram into a terminology that helps writing equations in the computer. The representation of the flow diagram involves classifying the parameters and variables defined in the model into stock variables (which are a mental photograph of the system), flow variables (elements determining the variation of levels), parameters and auxiliary variables (Table 1 and Fig 2 ). The equations used to model the SASM are formulated in Table 2 from the SD method perspective, a general notation has been considered. 
Thus, the strategies alignment process is modelled using the SD method. The simulation software used to represent the SASM in the SD simulation approach is AnyLogic [18]. The optimiser package included in AnyLogic software allows to obtain the best set of parameters solutions while the system generates multiple scenarios in the simulation tool. AnyLogic uses the built-in OptQuest optimizer to search for the best solution, given the objective function, constraints, requirements, and parameters (decision variables). Supports objective values that are based on experimentation through the General Replication Algorithm. The optimization process enables the finding of the optimal combination of decision variables that maximise the network performance (KPI_GLOBAL): (i) the units of strategies to activate $u_{-} S_{i s}$ and (ii) the time when to activate them $t_{-} S_{i s}$. The application of the SASM in the context of supply network disruptions will allow to support enterprises in the collaborative decision making process of selecting proactive strategies to be activated, in order to be aligned, with the main aim of reducing the loss of performance as well as the time and cost of recovery when a disruption occurs.

Table 1. Stock, Flow, Parameter and Auxiliary Variables in the SASM

\begin{tabular}{|c|c|c|}
\hline & Elements & Definition \\
\hline \multirow{10}{*}{ 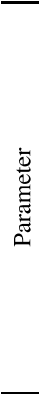 } & u_Sis & Units of strategy [u.s] Sis to be activated \\
\hline & tī_sis & Initial time of activation of Sis \\
\hline & c_s $\bar{S}$ is & Cost of the strategy \\
\hline & KPיIixk_min & Minimum increase that the enterprise estimates for the $K P I_{i x k}$ \\
\hline & Threshold_KPIixk & $\begin{array}{l}\text { Value from which the associated } K P I_{i x k} \text { is affected by the activation of a } \\
\text { strategy Sis }\end{array}$ \\
\hline & val_Sis_KPIixk & $\begin{array}{l}\text { Value that registers the increase or decrease of the } K P I_{i x k} \text { when one unit of } \\
\text { Sis is activated }\left(u_{-} \text {Sis }\right)\end{array}$ \\
\hline & Wikx & Relevance that the $K P I_{i x k}$ has for enterprise $i$ \\
\hline & d1_Sis & Delay time of activation of the strategy $s$ in enterprise $i$ Sis \\
\hline & d2_Sis & $\begin{array}{l}\text { Time between the Sis starts to influence the } K P I_{i x k} \text { until the maximum level } \\
\text { of influence in is achieved }\end{array}$ \\
\hline & d4_Sis & Total duration of Sis \\
\hline \multirow{7}{*}{ 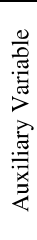 } & d3_Sis & $\begin{array}{l}\text { Time period in which Sis is exerting the highest influence } \\
\text { (val Sis KPIixk) on the } K P I_{i x k}\end{array}$ \\
\hline & Sis_mu & Monetary units invested in the activation of Sis \\
\hline & tf_sis & Time unit when Sis is finished [t.u.] \\
\hline & slope_Sis_KPIikx & Slope of the ramp in represented in curve_KPIixk \\
\hline & fulfill_KPIixk_min & Minimum increase that the enterprise estimates for the $K P I_{i x k}$, \\
\hline & KPI_i & Increase experienced by the KPI defined at enterprise level \\
\hline & KPI_GLOBAL & Increase experienced KPI defined at network level \\
\hline \multirow{3}{*}{$\frac{\sqrt{U}}{\stackrel{D}{\Delta}}$} & KPIixk & $\begin{array}{l}\text { Increase observed in the } K P I_{i x k} \text { when the Sis is activated: Sis activated in the } \\
\text { same enterprise (intra-enterprise) and } S j s \text { activated by other enterprises } \\
\text { (inter-enterprise) }\end{array}$ \\
\hline & KPIixk_T & Increase experienced by the $K P I_{i x k}$ once the Threshold_kpi $i_{i x k}$ is computed \\
\hline & bi & $\begin{array}{l}\text { Budget owned by the enterprise } i \text { to invest in the activation of the strategies } \\
\text { Sis }\end{array}$ \\
\hline \multirow{3}{*}{$\frac{3}{1}$} & curve_KPIixk & $\begin{array}{l}\text { Function that models the increase of } K P I_{i x k} \text { considering all the activated } \\
\text { strategies }\end{array}$ \\
\hline & curve_KPIixk_T & $\begin{array}{l}\text { Function that models the increase of } K P I_{i x k} \text { when Threshold_kpi } i_{i x k} \text { value is } \\
\text { computed }\end{array}$ \\
\hline & Inf_Sis_KPIixk & Function that models the behaviour of the $K P I_{i x k}$ when Sis is activated \\
\hline
\end{tabular}


Table 2. Equations for the SASM in SD

\begin{tabular}{|c|}
\hline $\begin{array}{l}\text { Dimensions } \\
\text { dimension_KPIixk, representing the indexes of the KPIs defined in the model index_KPIixk } \\
\text { dimension_Sis, representing the indexes of the strategies defined in the model index_Sis }\end{array}$ \\
\hline $\begin{array}{l}\text { Budget } \\
\text { bi }- \text { SSis mu }\end{array}$ \\
\hline $\begin{array}{l}\text { Monetary units invested in the activation of } s_{\text {is }} \\
\text { Sis_mu }=\text { u_Sis } \cdot \text { c_Sis.get (index_Sis) }\end{array}$ \\
\hline $\begin{array}{l}\text { Unit of time when } s_{t} \text { is finished } \\
\text { tf_Sis }=\text { ti_Sis }+ \text { d4_Sis.get }(\text { index_Sis) }\end{array}$ \\
\hline $\begin{array}{l}\text { Time period in which } s_{r_{i s}} \text { is exerting the highest influence }\left(i n f_{-} t_{r_{i s \_}} k p i_{i x k}\right) \text { on the } k p i_{i x k} \\
\text { d3_Sis = d4_Sis.get(index_Sis) - d1_Sis.get(index_Sis) - } \\
(2 \cdot \mathrm{d} 2 \text { _Sis.get(index_Sis) ) }\end{array}$ \\
\hline 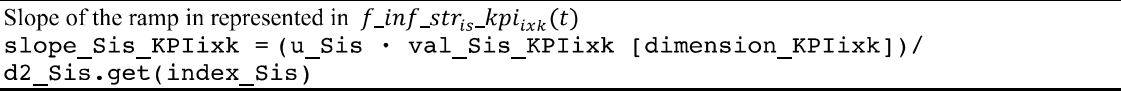 \\
\hline $\begin{array}{l}\text { Function that models the behaviour of the } k i_{i x k} \text { when } s_{\text {is }} \text { is activated } \\
\text { Inf_Sis_KPIixk }=\text { delay (ramp (slope_Sis_KPIixk[dimension_KPIixk], ti_Sis, } \\
\text { ti_Sis }+ \text { d2_Sis.get(index_Sis)) - ramp (slope_Sis_KPIixk[dimension_KPIixk], } \\
\text { ti_Sis }+ \text { d2_Sis.get(index_Sis) }+ \text { d3_Sis, ti_Sis }+2 \cdot \text { d2_Sis.get(index_Sis) } \\
+ \text { d3_Sis), d1_Sis.get(index_Sis)) }\end{array}$ \\
\hline $\begin{array}{l}\text { Function that models the overall behaviour of the } k p i_{i x k} \text { considering all the activated strategies } \\
\text { curve KPIixk }=\sum \text { Inf } \mathrm{S} 11 \text { KPIixk [dimension KPIixk] }\end{array}$ \\
\hline $\begin{array}{l}\text { Increase observed in the } k p i_{i x k} \\
\text { KPIixk }=\int \text { curve KPIixk[dimension KPIixk] }\end{array}$ \\
\hline $\begin{array}{l}\text { Function that models the curve of the behaviour of the } k_{i x i_{i x k}} \text { when the Threshold_kpi } i_{i x k} \text { value is rested } \\
\text { Curve_KPIixk_T }=\text { IF ((curve_KPIixk[dimension_KPIixk] >= } \\
\text { Threshold_KPIixk[dimension_KPIixk]) THEN (curve_KPIixk[dimension_KPIixk] - } \\
\text { Threshold_KPIixk[dimension_KPIixk]) ELSE (IF } \\
\text { (curve_KPIixk[dimension_KPIixk]<0) THEN curve_KPIixk[dimension_KPIixk] ELSE } \\
\text { ()) }\end{array}$ \\
\hline $\begin{array}{l}\text { Increase experienced by the } k p i_{i x k} \text { once the Threshold_kpi } i_{i x k} \text { is computed } \\
\text { KPIixk_T }=\int \quad \text { curve_KPIixk_T [ dimension_KPIixk ] }\end{array}$ \\
\hline $\begin{array}{l}\text { Acomplishment of the minimum increase that the enterprise determines for the } k i_{i x k} \text {, once the Threshold_kpi } i_{i x k} \\
\text { is computed } \\
\text { fulfill_KPIixk_min }=\text { IF ( }(\text { KPIixk_T [ dimension_KPIixk ] >= KPIixk_min [ } \\
\text { dimension_KPIixk ] ) THEN } 1 \text { ELSE } 0)\end{array}$ \\
\hline $\begin{array}{l}\text { Increase experienced by the KPI defined at enterprise } i \text { level } \\
\text { KPI_i }=\Sigma \text { KPIixk_T.get }(\text { index_KPixk) } \cdot \text { Wixk[dimension_KPIixk] }\end{array}$ \\
\hline $\begin{array}{l}\text { Increase experienced KPI defined at network net level } \\
\text { KPI GLOBAL }=\Sigma \mathrm{KPI} i / \mathrm{n}\end{array}$ \\
\hline
\end{tabular}

\section{Illustrative Example}

In this section, an illustrative example is presented in order to demonstrate the application of the SASM to deal with disruptive events and enhance resilience and agility in the $\mathrm{CN}$ and its enterprises. In this example, two enterprises are considered acquiring the roles of supplier (enterprise 1) and manufacturer (enterprise 2). Each enterprise defines two proactive strategies in order to deal with a production process disruption due to the interruption of material supply caused by a machine breakdown in the supplier plant (the supplier cannot provide the required products with the requirements specified by the focal company) [13]. 


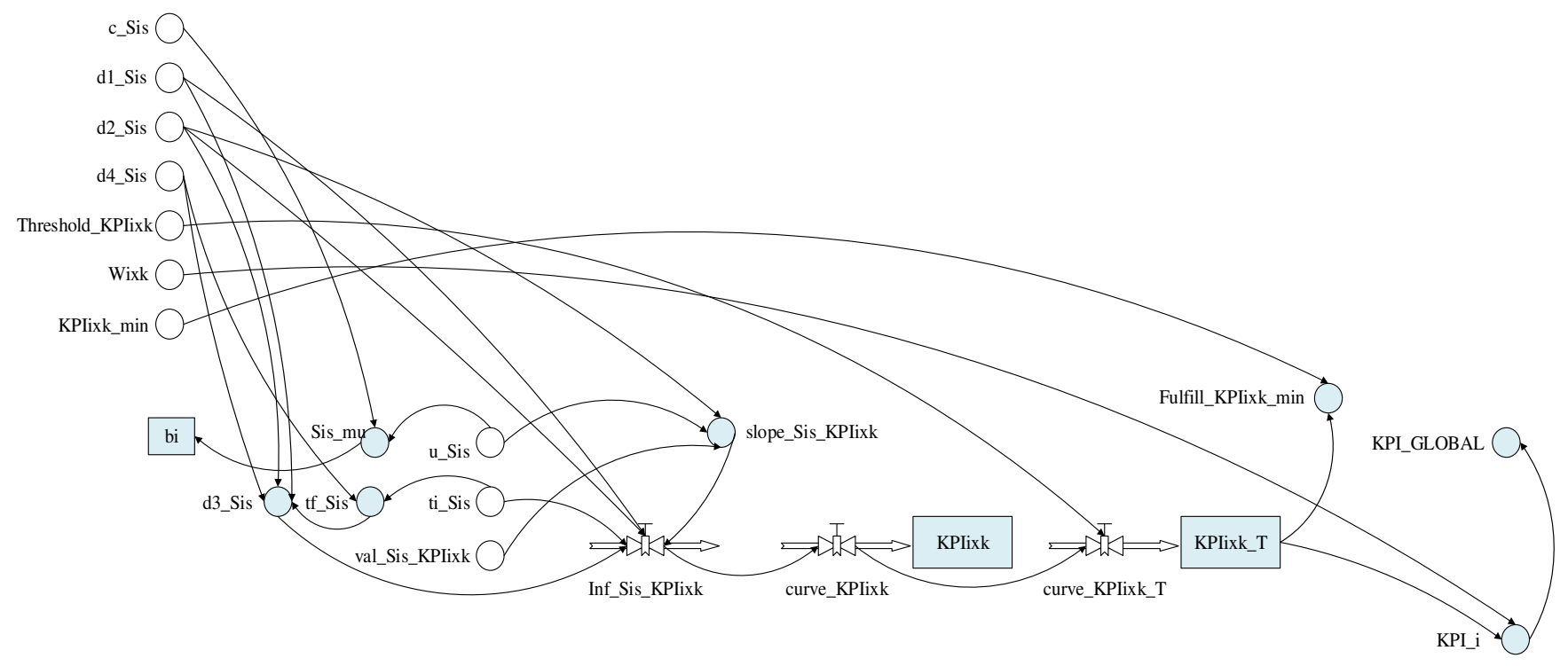

Fig. 2. SASM: Flow Diagram 
Supplier

- Strategy 1(S11): Increase the level of Safety Stock

- Strategy 2(S12): Total Productive Maintenance

Manufacturer

- Strategy 1(S21): Increase Suppliers Base

- Strategy 2(S22): Increase the level of Safety Stock

Moreover, each enterprise defines two KPIs to measure the influence that the strategies have on the recovery phase. These indicators are related to the cost and the time of recovery for each of the enterprises participating in the $\mathrm{CN}$. Accordingly $\mathrm{KPI}_{111}$ (of the supplier) and $\mathrm{KPI}_{211}$ (of the manufacturer) refer to the reduction of

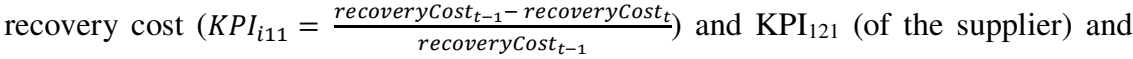
$\mathrm{KPI}_{221}$ (of the manufacturer) refer to the reduction of recovery time $\left(K P I_{i 21}=\right.$ $\frac{\text { recoveryTime }_{t-1}-\text { recoveryTime }_{t}}{\text { recoveryTime }_{t-1}}$. Table 3 shows all the data required by SASM. First, the budget that each enterprise owns to activate the proactive strategies is defined. Regarding the strategies characterisation, the activation cost of the strategies and the duration parameters are estimated by the enterprises. With respect to the performance indicators, the weight and the threshold values are given. Finally, the values of influence are estimated by each enterprise (Val_Sis_KPIixk).

Table 3. Illustrative Example: Data

\begin{tabular}{|c|c|c|c|c|c|c|c|c|c|c|c|c|c|c|c|}
\hline & & & & & & & & & & & & \multicolumn{2}{|l|}{$k p i_{111}$} & \multicolumn{2}{|l|}{$k p i_{121}$} \\
\hline & & & & & & & & & & & & $w_{111}$ & 0,5 & $w_{121}$ & 0,5 \\
\hline & & & & & & & & & & & & \begin{tabular}{|l} 
Threshold_kpi $i_{111}$ \\
\end{tabular} & 0,2 & Threshold_kpi $i_{121}$ & 0,1 \\
\hline \multirow{8}{*}{$\frac{S_{11}}{S_{12}}$} & $u_{-} S_{11}$ & $?$ & $t i S_{1 i}$ & $?$ & $c_{-} S_{11}$ & 1 & $d_{1} S_{11}$ & 0,05 & $d_{2} S_{11}$ & 0,01 & \begin{tabular}{l|l}
$d_{4} S_{21}$ & 0,3 \\
\end{tabular} & val_S $S_{11} k p i_{111}$ & 0,9 & val_s $S_{11} k p i_{121}$ & $-0,02$ \\
\hline & $u_{-} S_{12}$ & ? & $t i I_{1} S_{12}$ & $?$ & $c_{-} S_{12}$ & 2 & $d_{1} s_{12}$ & 0,2 & $d_{2} s_{12}$ & 0,03 & \begin{tabular}{|l|l|}
$d_{4} S_{21}$ & 0,6 \\
\end{tabular} & val__s$s_{12-} k p i_{111}$ & 0,5 & val__ $s_{12} k p i_{121}$ & 0,4 \\
\hline & & & & & & & & & & & & val__$S_{21-} k p i_{111}$ & $-0,8$ & val__ $S_{21-} k p i_{121}$ & $-0,4$ \\
\hline & & & & & & & & & & & & val__sz $S_{2 z} k p i_{111}$ & 0,8 & val_s $S_{22} k p i_{121}$ & 0,8 \\
\hline & & & & & & & Manufact & urer 4 & $b 2=6$ & & & & & & \\
\hline & & & & & & & & & & & & $k p i_{211}$ & & $k p i_{221}$ & \\
\hline & & & & & & & & & & & & $w_{211}$ & 0,5 & $w_{221}$ & 0,5 \\
\hline & & & & & & & & & & & & $\mid$ Threshold_kpi $i_{211}$ & 0,3 & \begin{tabular}{|l} 
Threshold_kpi $i_{221}$ \\
\end{tabular} & 0,15 \\
\hline$S_{21}$ & $u_{-} S_{21}$ & $?$ & $t i_{-} S_{21}$ & $?$ & $c_{-} S_{21}$ & 5 & $d_{1} S_{21}$ & 0,05 & $d_{2} S_{21}$ & 0,02 & \begin{tabular}{l|l}
$\boldsymbol{d}_{4} \boldsymbol{S}_{21}$ & 0,75 \\
\end{tabular} & val__ $S_{21-} k p i_{211}$ & 1 & \begin{tabular}{|l} 
val $S_{21}$ kppi \\
221
\end{tabular} & 0 \\
\hline$S_{22}$ & $u_{-} S_{2 z}$ & $?$ & $t i i_{22}$ & $?$ & $c_{-} S_{z z}$ & 6 & $d_{1} s_{21}$ & 0,1 & $d_{2} S_{21}$ & 0,01 & \begin{tabular}{|l|l|}
$d_{4} s_{21}$ & 0,5 \\
\end{tabular} & val_s $S_{22} k_{p i} i_{211}$ & 0,8 & val__s2_kpi S $_{221}$ & 0,8 \\
\hline & & & & & & & & & & & & val__s $\mathrm{S}_{11} \mathrm{kpi}_{211}$ & $-0,7$ & val_S $S_{11} k p i_{221}$ & $-0,2$ \\
\hline & & & & & & & & & & & & val_S $S_{12}$ kpi $_{211}$ & 0,8 & val_S $S_{12}$ kpi & 0,8 \\
\hline
\end{tabular}

In this case, the collaborative scenario modelled takes into account all the values of influence. Considering the data provided and introducing this data in the SASM simulation software (AnyLogic) in which the SASM is modelled, the optimisation experiment is done to obtain the decision variables that maximise the network performance level (KPI_GLOBAL). The results of the decision variables concerning (i) the units of strategies to activate $u_{-} S_{i s}$ and (ii) the time when to activate them $t i_{-} S_{i s}$ are shown in Fig. 3. The result of the collaborative scenario shows that, in order to have an efficient recovery and increase the levels of resilience and agility of the network, the supplier must activate the two defined strategies. The activation time of the supplier's strategies will be ti_S11 $=0.162$ and ti_S12 $=0.063$, considering that the unit refers to one year, strategy S11 will be initialised at the day $59(0,162 \cdot 365$ days $)$ and strategy S12 will be initialised at the day 23, from the beginning of the year. Whilst, the manufacturer must only activate the strategy S22 at the day 59'5. 


\begin{tabular}{|c|c|c|c|}
\hline & & Current & Best \\
\hline Iteration: & & 4,997 & 3,228 \\
\hline Objective: & $\uparrow$ & 0.501 & 0.501 \\
\hline
\end{tabular}

\begin{tabular}{|c|c|c|}
\hline \multicolumn{3}{|c|}{ Parameters } \\
\hline u_S11 & 1 & 1 \\
\hline ti_S11 & 0.162 & 0.162 \\
\hline u_S12 & 1 & 1 \\
\hline ti_S12 & 0.064 & 0.063 \\
\hline u_S21 & 0 & 0 \\
\hline ti_S21 & 0.201 & 0.199 \\
\hline u_s22 & 1 & 1 \\
\hline ti_S22 & 0.163 & 0.163 \\
\hline C_Sis & [ dimension_Scdith & sion_Sis ] \\
\hline
\end{tabular}

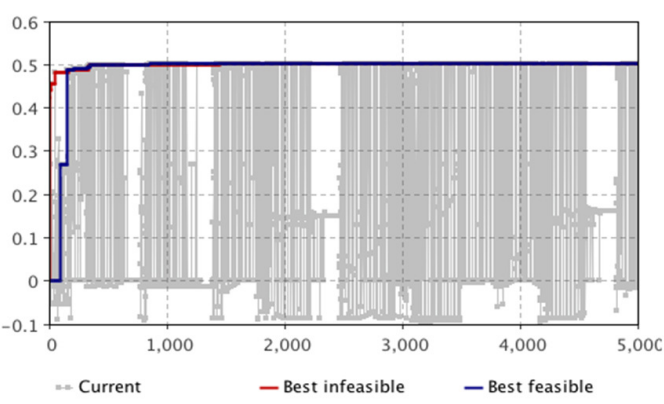

Fig. 3. SASM: Flow Diagram

Table 4 presents a comparison of the results obtained in the collaborative scenario with those obtained in the non-collaborative scenario, in both cases using the SASM. In the non-collaborative scenario the supplier only considers his own estimated values of influence, without considering how the strategies formulated by the manufacturer influence the performance level of his own indicators (shaded with grey in Table 3); the same happens to the manufacturer. In this regard, the non-collaborative scenario only considers intra-enterprise influences. The collaborative scenario is a more realistic one considering both intra and inter-enterprise influences, in which the supplier not only considers his own estimated values of influence, but also considers how the strategies formulated by the manufacturer influence the performance level of his own indicators, the same happens to the manufacturer.

From Table 4, it can be concluded that in the proposed illustrative example, the optimised solution of the collaborative scenario generates a level of network performance significantly higher than the performance resulting from the solution obtained in the non-collaborative scenario. Moreover, the solution obtained in the non-collaborative scenario breach the restriction of non-negativity values of KPI111 and KPI121. Whereas that the solution of the collaborative scenario complies with the non-negativity restriction so that all the performance indicators are larger than 0 . Making collaboratively decisions of which strategies to activate in order to deal with a certain disruption, using the SASM provides better solutions than if this decision is performed individually.

The SASM serves as a supporting tool, for enterprises participating in $\mathrm{CN}$, to deal with disruptive events, so that the activation of aligned proactive strategies involves an improvement in time and cost of recovery. Consequently, the application of SASM is an effective tool to increase the resilience and agility of $\mathrm{CN}$ in terms of identifying the aligned strategies that will allow dealing with potential disruptions. 
Table 4. SASM Comparison of the results: Non-Collaborative vs. Collaborative scenario

\begin{tabular}{|c|c|c|}
\hline & Non-collaborative Scenario & Collaborative Scenario \\
\hline$u \_S 11$ & 1 & 1 \\
\hline ti_S11 & 0,169 & 0,162 \\
\hline$u \_S 12$ & 1 & 1 \\
\hline$t i \_S 12$ & 0 & 0,063 \\
\hline$u \_S 2 I$ & 1 & 0 \\
\hline$t i \_S 21$ & 0,239 & 0,199 \\
\hline$u \_S 22$ & 0 & 1 \\
\hline$t i \_S 22$ & 0 & 0,163 \\
\hline KPIII1_T & $-0,195$ & 0,663 \\
\hline Fulfill_KPII11_min & 0 & 1 \\
\hline KPII21_T & $-0,139$ & 0,45 \\
\hline Fulfill_KPI121_min & 0 & 1 \\
\hline KPI211_T & 0,587 & 0,357 \\
\hline Fulfill_KPI211_min & 1 & 1 \\
\hline KPI221_T & 0,189 & 0,533 \\
\hline Fulfill_KPI221_min & 1 & 1 \\
\hline KPI_I(distributor) & $-0,167$ & 0,557 \\
\hline KPI_2 (manufacturer) & 0,388 & 0,445 \\
\hline KPI_GLOBAL & 0,1105 & $\mathbf{0 , 5 0 1}$ \\
\hline
\end{tabular}

\section{Conclusions}

This paper proposes the Strategies Alignment Simulation Model (SASM) for aligning the proactive strategies formulated in order to reduce the recovery time and cost in the case of a process disruption, in the $\mathrm{CN}$ context. It has been proved, in the illustrative example, that deciding about the activation of aligned strategies from a collaborative perspective provides better results in terms of disruption recovery time and cost. Ultimately, the results obtained using the SASM allow increasing the resilience and agility of the $\mathrm{CN}$. The main drawback considered is the data collection, especially with respect to the values of influence ( $v a l \_S i s \_$KPIixk), which the enterprises have to estimate. As the enterprise may face up to a disruption that has never occurred, this estimation could become very difficult. In the light of the results obtained, future research lines lead to propose guidelines to support enterprises on the data gathering and sharing along the strategies alignment process. In the collaborative scenario, the exchange of information is considered a key factor; therefore, future work will be also devoted to enhance the information sharing process. Finally, the SASM will be applied in a real case study in order to obtain the proper feedback from the enterprises and improve the simulation model.

Acknowledgments. This work was funded in part by the European Community's 7FP programme (FP7/2007-2013) under the grant agreement $n^{\circ}$ NMP2-SL-2009229333 and the Programa Val $i+d$ para investigadores en formación (ACIF) 


\section{References}

[1] Camarinha-Matos, L.M. and Afsarmanesh, H.: Collaborative networks: a new scientific discipline. J. Intell. Manuf., vol. 16, no. 4-5, pp. 439-452 (2005).

[2] Bititci, U., Turnera, T., Mackaya, D., Kearneyc, D., Parunga, J. and Waltersb, D.: Managing synergy in collaborative enterprises. Prod. Plan. Control Manag. Oper., vol. 18, no. 6, pp. 454-465 (2007).

[3] Macedo, P., Abreu, A., and Camarinha-Matos, L.M.: A method to analyse the alignment of core values in collaborative networked organisations. Prod. Plan. Control, vol. 21, no. 2, pp. 145-159, Mar (2010).

[4] Andres, B. and Poler, R.: Computing the Strategies Alignment in Collaborative Networks, in Enterprise Interoperability VI, K. Mertins, F. Bénaben, R. Poler, and J.-P. Bourrières, Eds. Cham: Springer International Publishing, pp. 29-40 (2014).

[5] Shamsuzzoha, A. H. M., Kankaanpaa, T., Helo, P., Carneiro, L.M., Almeida, R. and Fornasiero, R.: Non-hierarchical Collaboration in Dynamic Business Communities, in Collaborative Networks for a Sustainable World, L. M. Camarinha-Matos, X. Boucher, and H. Afsarmanesh, Eds. Springer Berlin Heidelberg, pp. 609-618 (2010).

[6] B Andres, B. and Poler, R.: Enhancing Enterprise Resilience through Enterprise Collaboration. IFAC Proc. Vol., vol. 7, no. 1, pp. 688-693 (2013).

[7] Sheffi, Y. and Rice, J. B.: A Supply Chain View of the Resilient Enterprise. A Supply Chain View of the Resilient Enterprise. MIT Sloan. Manag. Rev., vol. 47, no. 1, pp. 41-48 (2005).

[8] Chorn, N. H.: The alignment theory: creating strategic fit. Manag. Decis., vol. 29, no. 1, pp. 20-24 (1991).

[9] Mitroff, I. and Alpasan, M.: Preparing for the evil. Harv. Bus. Rev., pp. 109-115, (2003).

[10] Barroso, A.P., Machado, V. H., and Cruz-Machado, V.: Supply Chain Resilience Using the Mapping Approach, in Supply Chain Management, L. Pengzhong, Ed. InTech, pp. 161-184 (2011).

[11] Wu, T., Blackhurst, J. and O'grady, P.: Methodology for supply chain disruption analysis. Int. J. Prod. Res., vol. 45, no. 7, pp. 1665-1682 (2007).

[12] Ivanov, D., Sokolov, B. Dolgui, A. and Solovyeva, I.: Application of Control Theoretic Tools to Supply Chain Disruptions Management, in Proceedings of the IFAC Conference on Manufacturing Modelling, Management and Control, pp. 1926-1931 (2013).

[13] Sanchis, R., and Poler, R.: Enterprise resilience assessment: a categorisation framework of disruptions. Dir. y Organ., vol. 54, pp. 45-53 (2014).

[14] Sanchis R. and Poler, R.: Definition of a Framework to Support Strategic Decisions to Improve Enterprise Resilience. IFAC Proc. Vol., vol. 7, no. 1, pp. 700-705 (2013).

[15] Andres, B. and Poler, R.: Dealing with the Alignment of Strategies within the Collaborative Networked Partners. IFIP Int. Fed. Inf. Process., vol. 450, pp. 13-21, (2015).

[16] Forrester, J. W.: Industrial dynamics. Cambridge, MA: MIT press (1961).

[17] Campuzano, F. and Mula, J.: Supply Chain Simulation. A System Dynamics Approach for Improving Performance. Springer London Dordrecht Heidelberg New York, p. 106 (2011).

[18] AnyLogic, “AnyLogic ® Available: http://www.anylogic.com/ (2015). 